\title{
Rod like attapulgite/poly(ethylene terephthalate) nanocomposites with chemical bonding between the polymer chain and the filler
}

\author{
L. Chen, K. Liu, T. X. Jin, F. Chen, Q. Fu* \\ Department of Polymer Science and Materials, State Key Laboratory of Polymer Materials Engineering, Sichuan \\ University, 610065 Chengdu, China
}

Received 7 December 2011; accepted in revised form 16 February 2012

\begin{abstract}
Poly(ethylene terephthalate) (PET) nanocomposites containing rod-like silicate attapulgite (AT) were prepared via in situ polymerization. It is presented that PET chains identical to the matrix have been successfully grafted onto simple organically pre-modified AT nanorods (MAT) surface during the in situ polymerization process. The covalent bonding at the interface was confirmed by Fourier transform infrared spectroscopy (FTIR) and thermogravimetric analysis (TGA). The content of grafted PET polymer on the surface of MAT was about $26 \mathrm{wt} \%$. This high grafting density greatly improved the dispersion of fillers, interfacial adhesion as well as the significant confinement of the segmental motion of PET, as compared to the nanocomposites of PET/pristine AT (PET/AT). Owing to the unique interfacial structure in PET/MAT composites, their thermal and mechanical properties have been greatly improved. Compared with neat PET, the elastic modulus and the yield strength of PET/MAT were significantly improved by about 39.5 and $36.8 \%$, respectively, by incorporating only 2 wt \% MAT. Our work provides a novel route to fabricate advanced PET nanocomposites using rod-like attapulgite as fillers, which has great potential for industrial applications.
\end{abstract}

Keywords: polymer composites, poly(ethylene terephthalate), attapulgite, interfacial structure, mechanical property

\section{Introduction}

Poly(ethylene terephthalate) (PET) is a semicrystalline polymer with excellent chemical resistance, thermal stability, and spinnability. Due to its low cost and high performance, PET is in widespread use in our modern life, ranging from textile fibers, films, bottles containers and food packaging materials to engineering plastics, etc. However, numerous shortcomings such as low rate of crystallization, long cycle times for injection moulding, low melt strength and low distortion temperature limit its application $[1,2]$. To meet the practical needs and improve its performance, the addition of inorganic filler into PET matrix has been a research focus, and dramatic enhancements in properties such as their mechanical, gas barrier property and thermal property can be achieved [3-13]. These enhancements result from homogeneous dispersion of nanoparticles in polymer matrix and the interaction between the nanoparticles and PET matrix. The in situ polymerization technique is particularly attractive, which enables to exercise control over both the polymer architecture and the final structure of the composites.

Recently various nanoparticles have been applied in the preparation of PET nanohybrids via in situ polymerization or melt mixing, including layered silicates montmorillonite (MMT) [3-6], spherical silica $[7,8]$, carbon nanotube [9-11] and so on. Specially, PET/layered clay composites have attracted much

\footnotetext{
${ }^{*}$ Corresponding author, e-mail: qiangfu@scu.edu.cn
}

(c) BME-PT 
attention. However, intercalation technology used in preparing PET/MMT composites makes the polymerization process complicated and expensive. Moreover, obtaining high exfoliation level of MMT in PET matrix still remains a great challenge.

Attapulgite (AT) is a crystalloid, hydrous magnesium-aluminum silicate mineral, which is received as a randomly oriented network of densely packed rods. A fibrillar single crystal is the structural unit, with $20-30 \mathrm{~nm}$ in diameter and $0.5-2.0 \mu \mathrm{m}$ in length [14-16]. Owing to the low price, relatively high surface area, good mechanical strength and thermal stability, AT is attracting more and more attention in the preparation of polymer/clay composites [1720]. The preparation of PET/AT has been also explored, and a silylation modification of AT was carried out via the reaction of silane coupling agents with the silanol groups on AT surface [21, 22]. In this route, the pre-modification was complicated and the improvement of mechanical properties was limited, moreover, the thermal stability of organic modifier must be taken into consideration.

In this work, we present a novel way to prepare PET composites using rod-like AT as filler, in which AT was first acid treated to produce more silanol groups on its surface, then 4, 4'-methylenebis(phenyl isocyanate) (MDI) was grafted on AT surface. Nanorods of raw AT and MDI modified AT (MAT) were incorporated into the PET matrix by Figure polymerization. It was assumed that during the in situ polymerization process, the high reactivity isocyanate groups on MAT surface can react with ethylene glycol followed by PET chain growth, resulting in the formation of PET chains identical to the polymer matrix grafted on MAT nanorods surface. It is demonstrated that PET chains are linked on MAT surface through covalent bond, which improves the dispersion and interface adhesion between MAT and PET matrices, leading to a greatly improvement of PET properties.

\section{Experimental section \\ 2.1. Materials}

Attapulgite clay (purity greater than $95 \%$, specific surface area $400-500 \mathrm{~m}^{2} / \mathrm{g}$ ) was supplied by Autobang International Co. (Xuyi County, Jiangsu, China). Terephthalic acid (TPA), 4, 4'-methylenebis (phenyl isocyanate) (MDI) were purchased from commercial sources. Ethylene glycol (EG), $\mathrm{Sb}_{2} \mathrm{O}_{3}$, triphenyl phoshite (TPP), hydrochloric acid, acetone, phenol, tetrachloroethane, trifluoroacetic acid, and dichloromethane were purchased from Kelong Co. (Chengdu, China), all reagents were used as received.

\subsection{Functionalization of AT nanorod}

AT nanorod was firstly activated for $1 \mathrm{~h}$ in $5 \mathrm{M} \mathrm{HCl}$ solution at $80^{\circ} \mathrm{C}$ to introduce more active hydroxyl groups on the surface. After exhaustive washing with deionized water to $\mathrm{pH}$ neutrality, the activated nanorod was dried and ground. Then it was re-dispersed it in dry acetone to form a $5 \mathrm{wt} \%$ suspension, excess MDI was added to the suspension with vigorous stirring and ultrasonic treatment. The mixture was refluxed at $80^{\circ} \mathrm{C}$ for $1 \mathrm{~h}$ to complete the reaction between isocyanate and hydroxyl groups. The grafted nanorod was centrifuged at $4000 \mathrm{rpm}$, and washed for 4-5 times with acetone to completely remove ungrafted MDI, then dried in a vacuum oven at $70^{\circ} \mathrm{C}$ in vacuo for $24 \mathrm{~h}$. The dried cake was ground and screened through a 200 mesh sieve to obtain MDI grafted nanorod (MAT).

\subsection{Preparation of PET composites}

PET/AT and PET/MAT composites were prepared by in situ polymerization. A given amount of MAT was first mixed with EG (124 mL, $2.25 \mathrm{~mol})$. Then the suspension was poured into a home-made autoclave together with TPA ( $249 \mathrm{~g}, 1.5 \mathrm{~mol}), \mathrm{Sb}_{2} \mathrm{O}_{3}$ and TPP $\left(\mathrm{Sb}_{2} \mathrm{O}_{3}\right.$ as catalyst, TPP as stabilizer). With stirring, the temperature was gradually increased to $100^{\circ} \mathrm{C}$, kept at this temperature for $30 \mathrm{~min}$ to let the remaining high reactivity isocyanate groups on MAT surface react with hydroxyl group of EG. Then the mixture was heated under reflux for $2 \mathrm{~h}$ at $230^{\circ} \mathrm{C}$ for direct esterification. After excess EG and water were distilled off, the mixture was heated to $280^{\circ} \mathrm{C}$ for condensation polymerization. The production was extruded from the autoclave by compressed nitrogen and cut into pellets. The pure PET and PET/AT were also prepared by the same method. PET composites with 1, $2 \mathrm{wt} \%$ AT and 1, $2 \mathrm{wt} \%$ MAT loading were labeled PET/1AT, PET/2AT, PET/1MAT and PET/2MAT, respectively. Finally, samples for mechanical test were obtained by meltpressing the pellets at $265^{\circ} \mathrm{C}$ and $5.0-8.0 \mathrm{MPa}$ 
between two iron flats using a hot-press, followed by quenching into ice-water to obtain a film without crystallization.

To investigate the interfacial structure and to estimate the grafting yield of PET/MAT, successive centrifugation/redissolution cycles [7] were employed to separate the nanorod from physically absorbed polymer. PET/2MAT (30 g) were dissolved in $150 \mathrm{~mL}$ mixture of phenol and tetrachloroethane $(1 / 1, w / w)$. The solution was centrifuged at $11000 \mathrm{rpm}$ for $30 \mathrm{~min}$, redissolved the obtained centrifugate in the above mixed solvent, and then separated again by centrifugation. This centrifugation/ redissolution procedure was repeated 5 times. The resulting solid materials were washed extensively with ethanol to remove the solvent and dried at $80^{\circ} \mathrm{C}$ under vacuum for $24 \mathrm{~h}$. AT was also separated from PET/2AT composites by the same procedure. The filler from PET/2MAT and PET/2AT was labeled IS-MAT and IS-AT, respectively.

\subsection{Characterization}

The intrinsic viscosity $([\eta])$ was determined at $25^{\circ} \mathrm{C}$ in an Ubbelohde viscometer with the mixture of 50/50 (wt/wt) phenol/1,1,2,2-tetrachloroethane as solvent. Prior to intrinsic viscosity test, the solution was filtered to remove the small amount of insoluble components.

FTIR was employed to characterize chemical structure of various nanorod. The sample was coated on a $\mathrm{KBr}$ plate, and the spectra were recorded between 400 and $4000 \mathrm{~cm}^{-1}$ on a Nicolet-560 FTIR spectrometer. The thermal stability was studied by Thermal gravimetric analysis (TGA) test, conducted at WRT-2P Shanghai Scale Factory, from 50 to $800^{\circ} \mathrm{C}$ at nitrogen atmosphere with the heating of $20^{\circ} \mathrm{C} / \mathrm{min}$. For SEM investigation, PET nanocomposites samples were cryo-fractured in liquid nitrogen and investigated on an Inspect SEM instrument (FEI) with an acceleration voltage of $20 \mathrm{kV}$.

The thermal analysis of the samples was conducted using a PerkinElmer DSC Pyris-1. Under nitrogen atmosphere, the samples of about $5.0 \mathrm{mg}$ were heated from room temperature to $300^{\circ} \mathrm{C}$ and held for $5 \mathrm{~min}$ to eliminate the thermal history, then cooled down to $30^{\circ} \mathrm{Cand}$ heated again at the rate of $10^{\circ} \mathrm{C} / \mathrm{min}$.

Dynamic Mechanical Analysis (DMA) test were conducted on a TA Company Q800, in tensile mode. Tests were performed at a frequency of $1 \mathrm{~Hz}$ with a temperature sweep from 40 to $120^{\circ} \mathrm{C}$ at a ramp rate of $3^{\circ} \mathrm{C} / \mathrm{min}$. Static force of $0.01 \mathrm{~N}, 25.0 \mu \mathrm{m}$ of setting amplitude, $135 \%$ of force track were applied to the samples with size of on samples $15.3 \mathrm{~mm} \times$ $5.5 \mathrm{~mm} \times 0.4 \mathrm{~mm}$. A total of 3 tests were performed for each batch of material. Tensile tests were performed using an universal tensile testing machine (Instron 4302, USA) equipped with a $500 \mathrm{~N}$ load cell, with a cross-head speed of $10 \mathrm{~mm} / \mathrm{min}$ at $23^{\circ} \mathrm{C}$. The tensile samples were cut from the hot-pressed films, with dimensions of $50 \times 5 \times 0.4 \mathrm{~mm}$. At least five samples were used for each measurement and the reported stress-strain curves below represent an average result.

\section{Results and discussion \\ 3.1. The interaction between PET and attapulgite}

It is well known that the enhanced performance of polymer/clay nanocomposites originate from the nanoscale dispersion of these inorganic fillers and the interaction between fillers and polymer matrix. AT is highly anisotropic clay which is typically agglomerated in the as-received condition, thus appropriate organic modification is a crucial point in the design and preparation of polymer/AT nanocomposites. In this work, an aromatic diisocyanate (MDI) was used to capture hydroxyl groups on AT surface, and then the MDI-grafted AT (MAT) were introduced to the PET matrix via in situ polymerization. This AT organic modification and composites synthesis process allows PET chains get linked on the MAT nanorod surface forming a novel polymer grafting interface structures, which can be confirmed by FTIR spectroscopy, TGA test and sedimentation experiment as discussed below.

Figure 1 shows the FT-IR spectra of neat PET, AT, IS-AT, MAT and IS-MAT. The spectrum for the original AT (Figure 1b) reveals characteristic absorption bands at $3500 \mathrm{~cm}^{-1}$ (from O-H stretching) and $1030 \mathrm{~cm}^{-1}$ (for $\mathrm{Si}-\mathrm{O}$ in-plane stretching). There is no much difference between spectra of raw AT and IS-AT(Figure 1c), except that there is a weak band at $1720 \mathrm{~cm}^{-1}$ that is attributed to the stretching resonance of PET carbonyl groups (Figure 1a), which indicates that there is still a little physically absorbed PET on AT surface that cannot be removed completely. While, in comparison with the spectrum of raw $\mathrm{AT}$, the characteristic absorption for isocyanate 


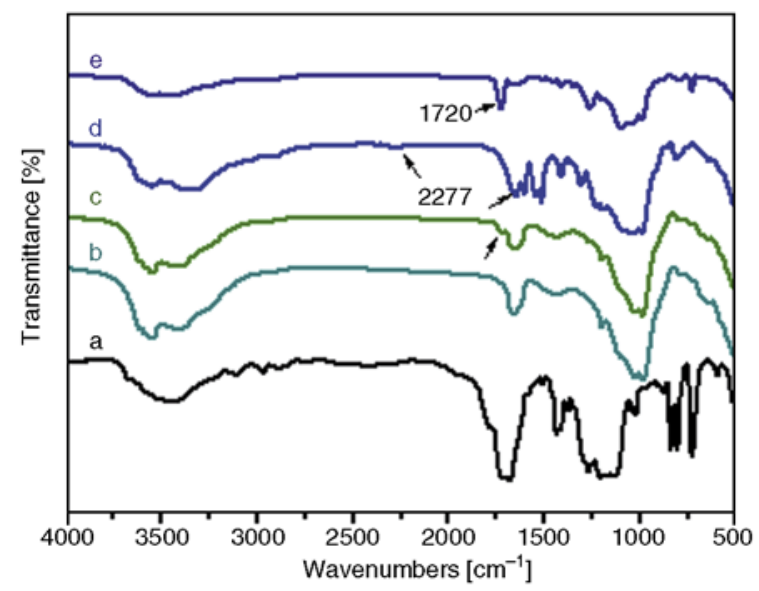

Figure 1. FTIR spectra for the (a) neat PET, (b) pristine AT, (c) IS-AT (AT isolated from PET/2AT nanocomposites), (d) MAT, and (e) IS-MAT (AT isolated from PET/2MAT nanocomposites)

groups at $2277 \mathrm{~cm}^{-1}$ is clearly identified in the spectrum of MAT (Figure 1d); urethane bands at 1648, 1511 , and $1309 \mathrm{~cm}^{-1}$, assigned to $\mathrm{CO}$ (urethane I), $\mathrm{NH}$ (urethane II), and CNH (urethane III), respectively, are also clearly identified. These characteristic bands indicate a covalent link between the isocyanate molecules and the AT. For the FTIR spectra of IS-MAT (Figure 1e), the characteristic absorption of $\mathrm{C}=\mathrm{O}$ stretching vibration in the ester carbonyl group at $1720 \mathrm{~cm}^{-1}$ is very strong compared with Figure 1b, the bands at 1579, 1409 and $729 \mathrm{~cm}^{-1}$ are due to in-plane and out-of-plane vibrations of the benzene rings, and the bands around 1263 and $1100 \mathrm{~cm}^{-1}$ result from vibrations of the ester group (Figure 1a). Since both IS-MAT and ISAT are prepared by the same procedure, these results verified that some PET molecules had been grafted onto MAT surface through chemical bonding rather than physical absorption. The proposed mechanism for the grafting polymerization is illustrated in Figure 2.

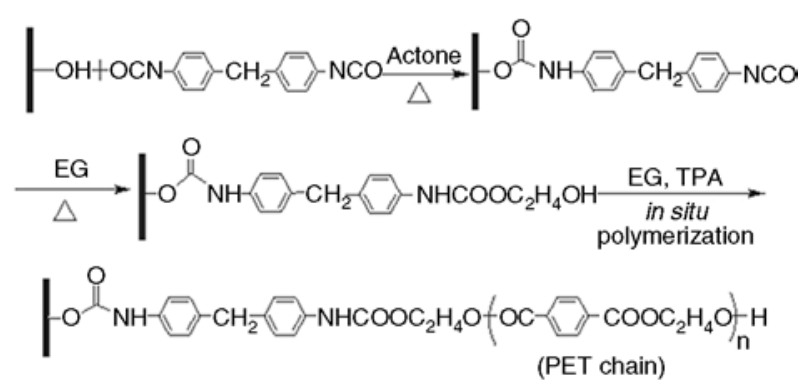

Figure 2. The reaction route of surface functionalization of AT and synthesize of PET nanocomposites by in situ polymerization in the presence of MAT nanorod

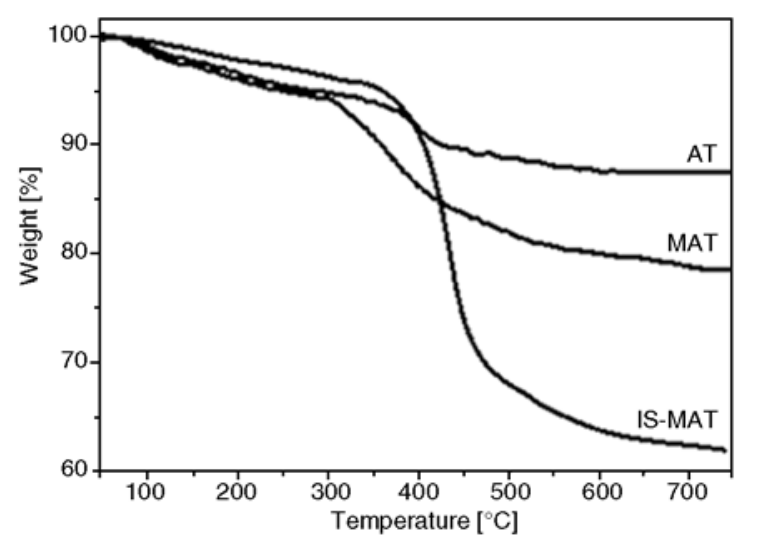

Figure 3. TGA curves of as-received AT; surface grafting with MDI (MAT); isolated nanorods from PET/ 2MAT composites (IS-MAT)

The amount of diisocyanate and PET polymer chains grafted onto the AT surface has been determined by thermogravimetric analysis (TGA) test. The weight loss curves for AT and MAT are presented in Figure 3. By extracting the mass loss of AT, we can calculate the mass fraction of organic component. Analysis of the curves indicates that $8.9 \mathrm{wt} \%$ (relative to the weight of dry original nanoparticles) of the MDI molecules has been grafted onto the surface of the nanorod, and about $26 \mathrm{wt} \%$ PET polymer has been grafted on MAT during the in situ polymerization. Compared with the amount of grafted MDI mass, the grafting PET polymer mass is relatively low, that may because the covalent graft PET chains on the surface of nanorod decreases the mobility of reactive end-groups, which reduces the opportunity to react in the polycondensation process and results in low molecular weight of grafted PET chains [7]. The aromatic structure of the grafting molecules makes the MAT exhibiting a higher onset temperature of thermal decomposition (near $300^{\circ} \mathrm{C}$ ). This is necessary for the in situ preparation of PET nanocomposites which is carried out at high temperature $\left(280^{\circ} \mathrm{C}\right)[4,23]$.

Finally, the evidence for improved dispersion of MAT in PET matrix and the interfacial bonding between the MAT and the PET can be further proved by examining the freeze-fractured cross-sections surfaces of PET/2AT and PET/2MAT composite. Figures $4 a, 4 b$ show the fracture surfaces of the PET composites loaded with $2 \mathrm{wt} \%$ raw AT, the nonuniform dispersion of AT that agglomerated massively in bundles in PET matrix can be clearly observed. Due to the lack of strong bonds holding 


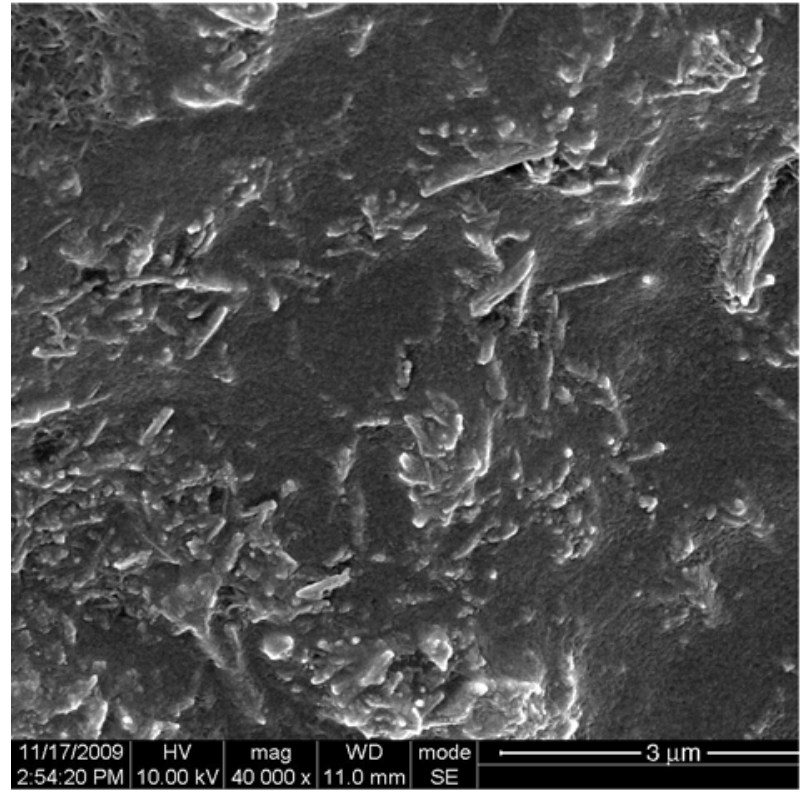

a)

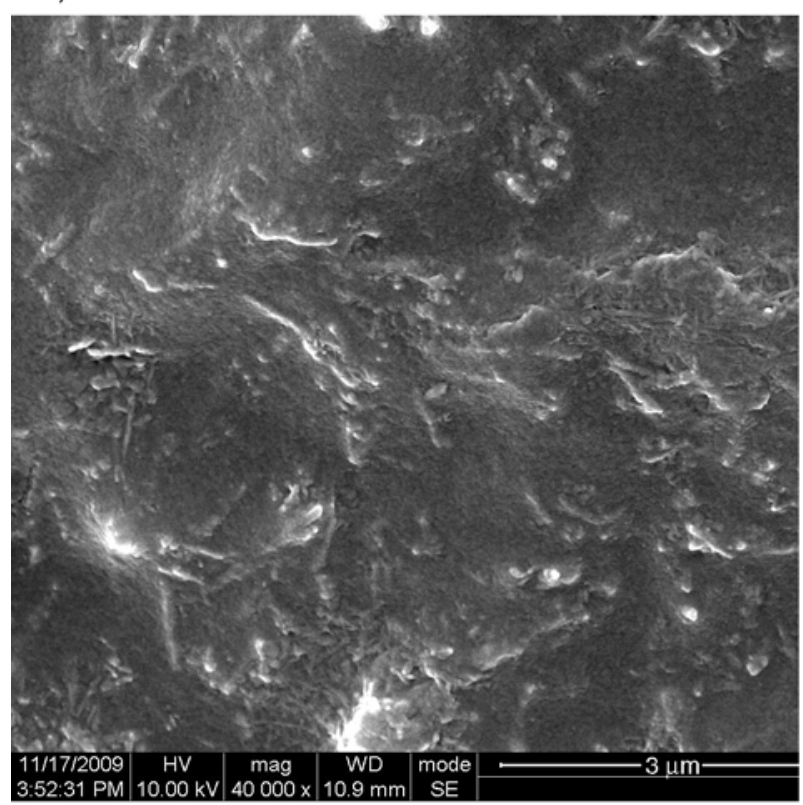

c)

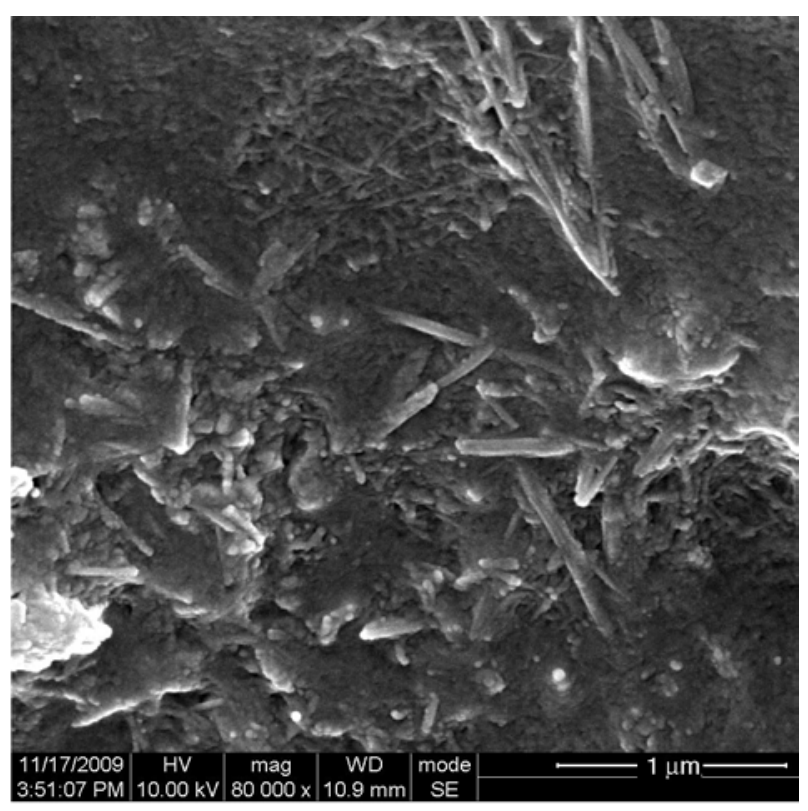

b)

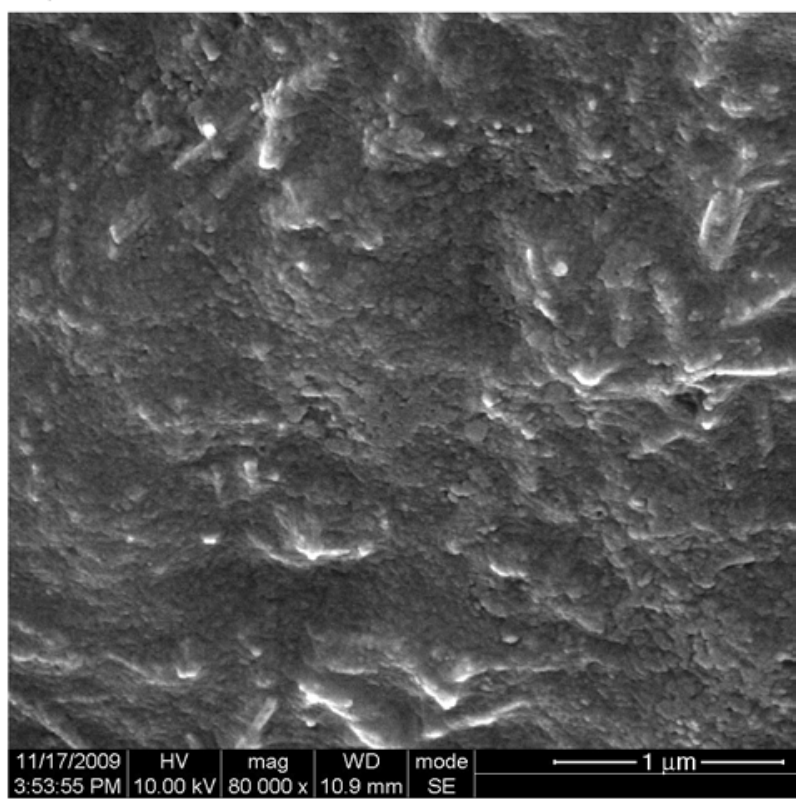

d)

Figure 4. SEM images of (a, b) PET/AT, (c, d) PET/MAT nanocomposites with $2 \mathrm{wt} \%$ nanorods loading, scale bar is $3 \mu \mathrm{m}$ and $1 \mu \mathrm{m}$, respectively

the PET polymer at the AT surface, many debonded AT nanorods are visible at the amplified image (Figure $4 \mathrm{~b}$ ). In contrast to pristine AT, MAT can be homogeneously dispersed in PET matrix that individual MAT can be easily identified without aggregation, and the interfacial boundary between MAT and PET matrix is nearly indiscernible as shown in Figures $4 \mathrm{c}, 4 \mathrm{~d}$, suggesting that their surfaces are most likely coated by a layer of PET polymer. It can be demonstrate from SEM images that the polymer covalent grafted PET/MAT composites dramatically improves the nanorod dispersion and greatly enhance the interfacial adhesion between the nanorod and PET matrix.

\subsection{Thermal properties}

Generally, the introduction of inorganic fillers into polymer matrices can improve the thermal stability of polymer. The decomposition temperatures measured at $5 \%\left(T_{\mathrm{d}}\right)$ and residues at $600^{\circ} \mathrm{C}\left(R_{\mathrm{e}}\right)$ are summarized in Table 1, which indicates a moderate increase in the thermal stability of the nanocomposites. The thermal decomposition $\left(T_{\mathrm{d}}\right)$ of the PET nanocomposites were $3-7^{\circ} \mathrm{C}$ higher than that of 
Table 1. Thermal properties of neat PET and PET nanocomposites

\begin{tabular}{|l|c|c|c|c|c|c|c|}
\hline \multicolumn{1}{|c|}{ Sample } & $\begin{array}{c}\boldsymbol{\eta}_{\mathbf{0}} \\
{[\mathbf{d L} / \mathbf{g}]}\end{array}$ & $\begin{array}{c}\boldsymbol{\eta}^{*} \\
{[\mathbf{d L} / \mathbf{g}]}\end{array}$ & $\begin{array}{c}\mathbf{T}_{\mathbf{g}} \\
{\left[{ }^{\circ} \mathbf{C}\right]}\end{array}$ & $\begin{array}{c}\mathbf{T}_{\mathbf{c}} \\
{\left[{ }^{\circ} \mathbf{C}\right]}\end{array}$ & $\begin{array}{c}\mathbf{T}_{\mathbf{m}} \\
\left({ }^{\circ} \mathbf{C}\right)\end{array}$ & $\begin{array}{c}\mathbf{T}_{\mathbf{d}} \\
{\left[{ }^{\circ} \mathbf{C}\right]}\end{array}$ & $\begin{array}{c}\mathbf{R}_{\mathbf{e}} \\
{\left[\mathbf{6 6 5 0}^{\circ} \mathbf{C}, \mathbf{w t} \%\right]}\end{array}$ \\
\hline NPET & 0.73 & 0.61 & 81.1 & 182 & 247 & 404.7 & 11.4 \\
\hline PET/1AT & 0.69 & 0.54 & 83.0 & 190 & 246 & 407.8 & 14.2 \\
\hline PET/2AT & 0.74 & 0.56 & 83.3 & 192 & 246 & 410.2 & 14.9 \\
\hline PET/1MAT & 0.73 & 0.60 & 85.6 & 194 & 249 & 409.4 & 15.7 \\
\hline PET/2MAT & 0.70 & 0.62 & 86.1 & 197 & 251 & 412.0 & 17.3 \\
\hline
\end{tabular}

$\eta_{0}, \eta^{*}$ represent intrinsic viscosity of neat PET and PET/AT composites before and after hot-pressing respectively; $T_{\mathrm{g}}$ values were obtained from dynamic mechanical analysis (DMA); $T_{\mathrm{c}}$ and $T_{\mathrm{m}}$ values were obtained from differential scanning calorimetry (DSC) tests.

neat PET, the residue content of the PET nanocomposites at $600^{\circ} \mathrm{C}$ is larger than that of neat PET, and the greater the clay content is, the larger the residue content is. Compared with PET/AT, PET/MAT composites have enhanced thermal stability. The particle-matrix interfacial interaction and 'barrier effect', which limits volatilization of products and thermal transport during polymers decomposition, have been suggested as the two main reasons for the improved thermal stability in polymer nanocomposites [20]. Therefore, it is reasonable to assume that the better dispersion and stronger interfacial interaction for PET/MAT result in the enhanced thermal stability of PET/MAT, as compared with the neat PET and PET/AT.

The thermal stability was also investigated by comparing the change of intrinsic viscosity for neat PET, PET/AT and PET/MAT during hot-press forming. As shown in Table 1, an obvious decrease of the intrinsic viscosity can be observed for all samples during hot-press forming. Compared with neat PET or PET/MAT, a larger decrease for PET/AT indicates that it undergoes a stronger degradation. Similar phenomena are also reported in other polymer/clay systems $[24,25]$. It is believed that the

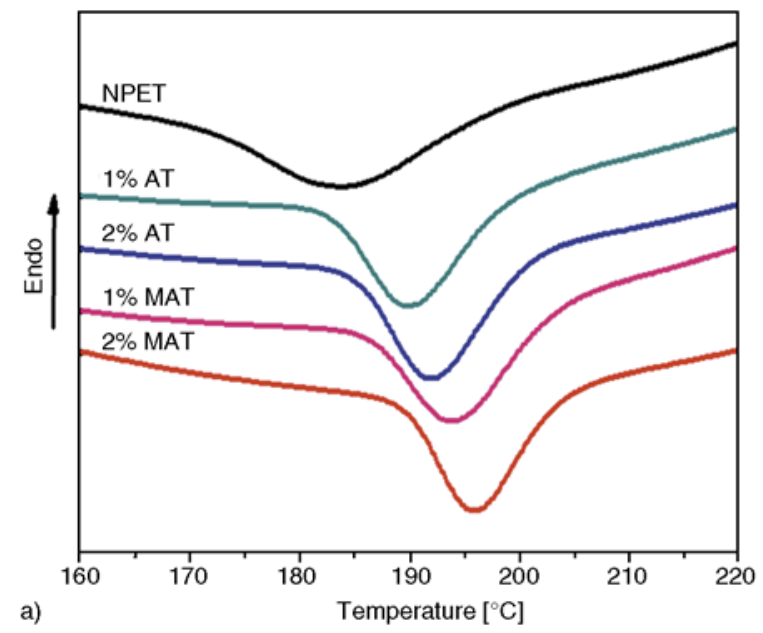

hydroxyl groups on the edge of clay platelet can act as Brønsted acidic sites to accelerate PET degradation [26]. AT is clay with a large amount of silanol group on the surface of nanorods compared with MMT, as the Figure 1a FTIR curve shows. That may be the main reason why PET/AT exhibited more severe degradation during hot-press process. For PET/MAT, MDI reacts with the hydroxyl groups on AT surface, and can reduce the amount of acidic sites. As a result, PET/MAT shows a slower degradation compared with PET/AT.

Pristine PET is a well-known semi-crystallization polymer with a character of low rate of crystallization. The effect of AT and MAT on PET crystallization and melting behaviors were examined by the DSC cooling and heating scans, respectively, as shown in Figure 5.

Figure 5a shows the crystallization curves of pure PET and PET nanocomposites, the $T_{\mathrm{c}}$ of PET differs among the samples and so does the width of the crystallization peak. One observes a remarkably increased $T_{\mathrm{c}}$, that is from $182^{\circ} \mathrm{C}$ of neat PET to 190 , $192^{\circ} \mathrm{C}$ of nanocomposites with $1,2 \mathrm{wt} \%$ AT, and to $194,197^{\circ} \mathrm{C}$ with $1,2 \mathrm{wt} \%$ MAT, respectively. The higher $T_{\mathrm{c}}$ and the narrower width of the crystalline

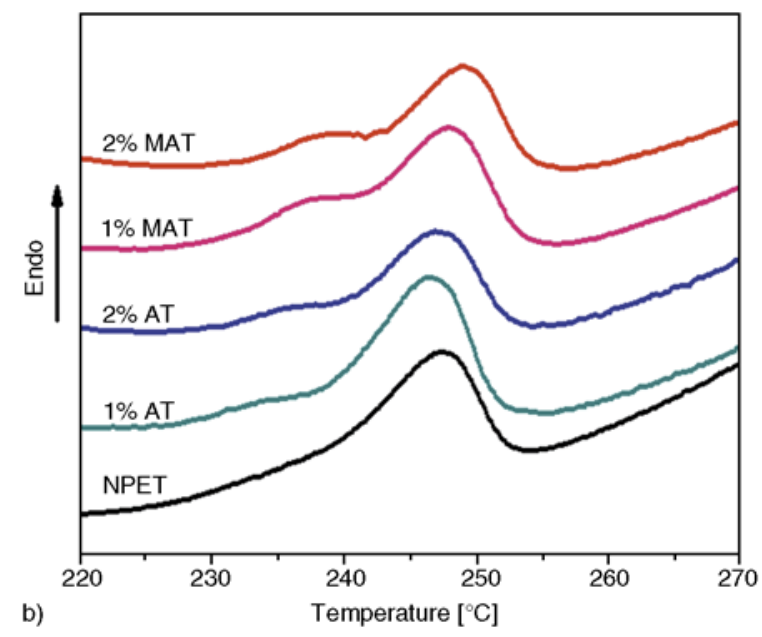

Figure 5. DSC curves for neat PET, PET nanocomposites during (a) cooling and (b) heating 
peaks observed in the nanocomposites can be explained by the nucleation effect of the nanorods on the PET [27]. It is demonstrated that both AT and functionalized MAT serve as nucleating agents for PET to accelerate the rate of crystallization, while, MAT seems to be a much more efficient nucleating agent for PET compared with AT. The apparent better nucleation effect of MATs in PET matrix is possibly due to the good dispersion of MAT in PET matrix, resulting in larger surface area serving as the nucleation sites. At the same time, the PET chains grafted on the surface of MAT nanorods via MDI can enhance the interaction with the PET matrix greatly. The two factors result in that MAT has a higher nucleating activity than AT and can further accelerate the crystallization rate of PET.

Figure $5 \mathrm{~b}$ depicts the heating runs of pure PET and PET nanocomposites. There are two melting endotherms that could be observed in each PET nanocomposite sample. It is suggested that the lower one represents the melting of imperfect or smaller crystals, and the higher one represents more perfect bigger crystallites $[28,29]$. It is interesting that with the content of AT or MAT increasing from 1 to $2 \mathrm{wt} \%$, the melting peak that is located at the low temperature becomes more distinct and increscent. Moreover, the melting peak $\left(T_{\mathrm{m}}\right)$ of the PET/AT sample located at higher temperature shows a little decrease compared with that of neat PET, in contrast, the PET/ MAT samples showed the reverse trend, whose melting peak showed $2-4^{\circ} \mathrm{C}$ increase.

The explanation of this phenomenon is that although the well-dispersed AT or MAT could act as nucleate agent to accelerated the process of PET crystallization, the growth of the crystallites would be restricted, resulting in imperfect or smaller crystals, and the

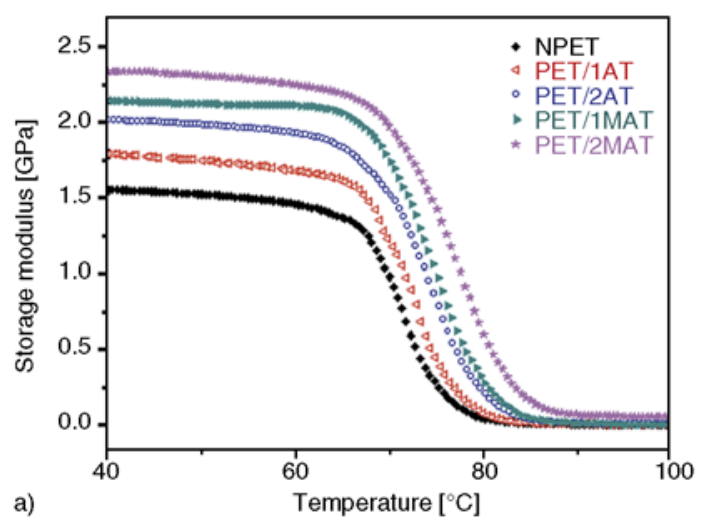

distribution of crystallites is broader than that of neat PET. With the increase of nanorods content, the spherulites become smaller and less perfect, so multi-melting peak shows up and become more visible, and the $T_{\mathrm{m}}$ of PET/AT decrease to lower temperature. However, the $T_{\mathrm{m}}$ of PET/MAT increased with the content of MAT and higher than that of neat PET. In this case, in the molten state, the segments of the PET molecules can easily interact with the nanorods with grafted PET chains on the surface, then crystallization nuclei developed, which results in some crystallites near the MAT nanorods surface. The intimate adhesion between MAT and PET matrix resulted in the decrease of PET chain mobility, which may have confined the movement of PET crystallite chains. Therefore, higher temperature is needed to provide chain segments with enough energy to start moving, and the melt temperature increases. This may be the main reason of the increases of $T_{\mathrm{m}}$ values in despite of smaller crystal size.

\subsection{Mechanical properties}

Particle networks and interfacial interactions are often cited as the two primary factors that dominate the mechanical properties of polymer nanocomposites [30]. Dynamic mechanical analysis (DMA) is an effective tool to characterize the interface of filled polymer systems, especially its damping spectra.

The dynamic storage modulus, $E^{\prime}$, are drawn in Figure $6 \mathrm{a}$ as a function of temperature. The $E^{\prime}$ value decreases with increasing temperature, and increases with nanorod content. It is clear that, the $E^{\prime}$ values of the PET/MAT are much higher than that of PET/AT composites over the entire temperature range. The

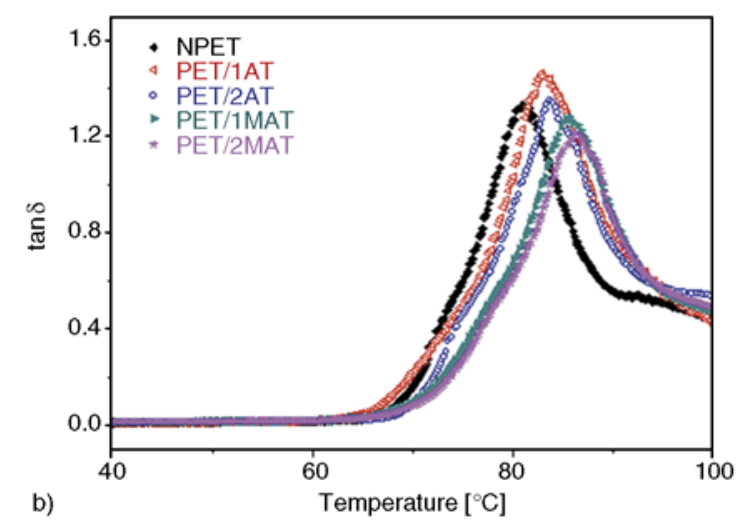

Figure 6. DMA diagrams for neat PET and PET nanocomposites. (a) curves of storage modulus versus temperature and (b) curves of $\tan \delta$ versus temperature. 
$E^{\prime}$ values of all samples at 40 and $80^{\circ} \mathrm{C}$ are also listed in Table 2 . The addition of nanorod significantly increases the value of $E^{\prime}$, and this increase is more obvious at higher temperatures. It indicates that the segmental motions of PET chains are restricted by the AT nanorod through physical or chemical interactions. Moreover, MAT with the good dispersion and interfacial interaction in PET/MAT significantly restricts the segmental movements, which results in higher $E^{\prime}$ values compared with PET/AT. By adding only $2 \mathrm{wt} \%$ of MAT, about $47 \%$ improvement of storage modulus has been achieved at $40^{\circ} \mathrm{C}$ compared with pure PET.

The DMA curves of the $\tan \delta$ versus temperature for all samples are presented in Figure 6b. Generally, The $T_{\mathrm{g}}$ values can be extracted from the peak in the $\tan \delta$ curves or loss modulus peak temperatures. The temperatures were extracted as $T_{\mathrm{g}}$ from the $\tan \delta$ curves in our study and listed in Table 1. It is interesting to note that the incorporation of AT lead to about $2^{\circ} \mathrm{C}$ increase of $T_{\mathrm{g}}$ values. While for the PET/ MAT system, a more obvious increase in $T_{\mathrm{g}}$ values can be found. It increases from $81.1^{\circ} \mathrm{C}$ for neat PET to $85.6^{\circ} \mathrm{C}$ for PET/1MAT and further increases to $86.1^{\circ} \mathrm{C}$ for PET/2MAT.

The glass transition temperatures of the polymer matrix depend on the free volume of the polymer. This temperature provides an indirect indication of the dispersion of nanoparticles in the matrix and the particle-matrix interaction, though other factors may also affect this transition. The enhanced $T_{\mathrm{g}}$ values reflects the strength of interfacial interaction and the microstructure of polymer nanocomposites $[9,20]$. From the $\tan \delta \sim T$ curves, the $T_{\mathrm{g}}$ of the PET/ AT shift slightly to higher temperature as AT loading increases which indicates that the pristine AT nanorods can limit the motion of the PET molecular chains in some extent. In terms of PET/MAT, the higher increase in $T_{\mathrm{g}}$ values indicate a stronger interfacial interaction between the MAT nanorod and the PET matrix. The direct attachment of PET chains onto MAT nanorod surface makes polymer chains more rigid, and their segmental movements are significantly restricted. With the increase of MAT content in PET films, the number of confined chains increases and therefore the larger elevation in $T_{\mathrm{g}}$ is a natural result.

The enhancement of mechanical properties can be also proved by the tensile testing. The stress-strain curves and mechanical properties of pure PET and the PET nanocomposites films are shown in Figure 7 and Table 2. For comparison, the composites reinforced with as-received AT are also included. Compare with pure PET, PET nanocomposites have higher tensile strength and tensile modulus. However, the enhancing effect is much pronounced in the case of the PET/MAT. The tensile strength of the PET/1MAT is significantly improved by $28 \%$ from 44.8 to $57.3 \mathrm{MPa}$, and the tensile modulus is improved by $17.8 \%$ from 1.57 to $1.85 \mathrm{GPa}$, relative to neat PET. While with $2.0 \mathrm{wt} \%$ MAT loading, the tensile strength and initial modulus are further enhanced to $62.5 \mathrm{MPa}$ and $2.14 \mathrm{GPa}$, which corresponds to a 39.5 and $36.8 \%$ enhancement compared to pure PET, respectively.

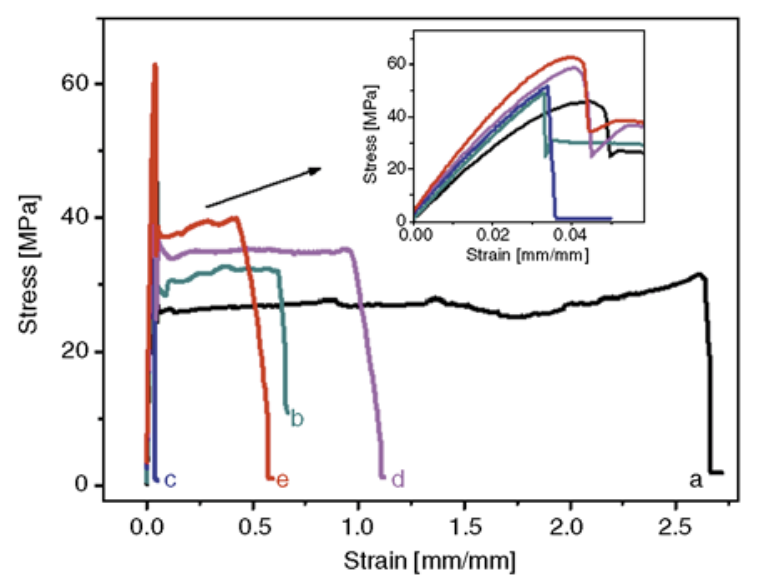

Figure 7. Stress-strain curves for (a) neat PET, (b) PET/ 1AT, (c) PET/2AT, (d) PET/1MAT and (e) PET/ 2MAT nanocomposites

Table 2. Mechanical properties from DMA and tensile test

\begin{tabular}{|c|c|c|c|c|c|}
\hline \multirow{2}{*}{ Sample } & \multicolumn{2}{|c|}{$\mathbf{E}^{\prime}$ [MPa] } & \multirow{2}{*}{$\begin{array}{c}\text { Tensile modulus } \\
\text { [GPa] }\end{array}$} & \multirow{2}{*}{$\begin{array}{c}\text { Tensile stress } \\
\text { [MPa] }\end{array}$} & \multirow{2}{*}{$\begin{array}{c}\text { Elongation at break } \\
{[\mathrm{mm} / \mathrm{mm}]}\end{array}$} \\
\hline & $40^{\circ} \mathrm{C}$ & $80^{\circ} \mathrm{C}$ & & & \\
\hline NPET & 1553 & 47 & $1.57 \pm 0.06$ & $44.8 \pm 1.7$ & $2.95 \pm 0.56$ \\
\hline \begin{tabular}{|l}
$\mathrm{PET} / 1 \mathrm{AT}$ \\
\end{tabular} & 1790 & 77 & $1.69 \pm 0.08$ & $48.3 \pm 2.1$ & $0.62 \pm 0.18$ \\
\hline PET/2AT & 2015 & 277 & $1.78 \pm 0.12$ & $50.6 \pm 3.0$ & $-^{*}$ \\
\hline PET/1MAT & 2140 & 307 & $1.85 \pm 0.07$ & $58.3 \pm 2.3$ & $1.16 \pm 0.34$ \\
\hline PET/2MAT & 2287 & 440 & $2.14 \pm 0.09$ & $62.5 \pm 2.8$ & $0.66 \pm 0.12$ \\
\hline
\end{tabular}

${ }^{*} \mathrm{PET} / 2 \mathrm{AT}$ composite shows a brittle-rupture tensile behavior, and its elongation at break is not listed. 
It has to be noted that the elongation at break for the PET nanocomposites decreased with the introduction of AT and MAT nanorod. PET/2AT composite shows a brittle-rupture tensile behavior, and its elongation at break is not listed in Table 2. It indicates that the nanocomposites became somewhat brittle compared with pure PET. As shown in Figure 7, PET/AT exhibits brittle-rupture behavior with $2 \mathrm{wt} \%$ loading, which results from the poor dispersion of pristine AT and the weak interfacial interaction between pristine AT and PET. However, PET/ MAT shows higher values of the elongation at break than that of PET/AT. By comparison, the higher value of the elongation at break of PET/ MAT should be attributed to the better dispersion of MAT nanorod and stronger interfacial bonding.

Many reported results have suggested that the formation of covalent bonding between nanoparticle fillers and polymer matrix, which lead to a more effective stress transfer, thus could improve the mechanical properties of polymer composites. For PET/MAT composites, the MAT nanorod is chemically bonded with PET chains, enabling a more efficient stress transfer from the PET matrix to the nanorod. It can be concluded that the good dispersion of MAT in the PET matrix and the strong interfacial adhesion lead to the significant enhancement of the mechanical properties of PET nanocomposites.

\section{Conclusions}

In this work, we presented a novel route to fabricate advanced PET nanocomposites using rod-like attapulgite as filler, where AT nanorod is chemically modified to be well dispersed in PET matrix and to possess a strong interaction with the matrix polymer. As confirmed by FTIR and TGA tests, the raw AT has been successfully functionalized in two steps: direct chemical attachment of MDI to the nanorod surface; then the isocyanate groups on surface of MAT nanorod react with EG during the in situ polymerization, resulting in PET chains grafted onto the MAT nanorod surface. The resulting interface structure is conducive for the dispersion of MAT nanorod and the increase of interfacial adhesion with the matrix polymer. The existence of MAT could enhance the thermal stability and accelerate the crystallization rate of PET. As expected, the high grafting density leads to efficient load transfer from the PET matrix to the nanorod, resulting in significant enhancement of the mechanical properties of the PET/MAT even having a small quantity of the MAT compare with pure PET or PET/AT.

\section{Acknowledgements}

We would like to express our great thanks to the National Natural Science Foundation of China for financial support (21034005, 51121001).

\section{References}

[1] Scheirs J., Long T. E.: Modern polyesters. Wiley, New York (2003).

[2] Fakirov S.: Handbook of thermoplastic polyesters. Wiley-VCH, Weinheim (2002).

[3] Tsai T-Y., Li C-H., Chang C-H., Cheng W-H., Hwang C-L., Wu R-J.: Preparation of exfoliated polyester/clay nanocomposites. Advanced Materials, 17, 1769-1773 (2005).

DOI: $\underline{10.1002 / \mathrm{adma} .200401260}$

[4] Chang J-H., Kim S. J., Joo Y. L., Im S.: Poly(ethylene terephthalate) nanocomposites by in situ interlayer polymerization: The thermo-mechanical properties and morphology of the hybrid fibers. Polymer, 45, 919926 (2004).

DOI: 10.1016/j.polymer.2003.11.037

[5] Pegoretti A., Kolarik J., Peroni C., Migliaresi C.: Recycled poly(ethylene terephthalate)/layered silicate nanocomposites: Morphology and tensile mechanical properties. Polymer, 45, 2751-2759 (2004).

DOI: $10.1016 /$ j.polymer.2004.02.015

[6] Costache M. C., Heidecker M. J., Manias E., Wilkie C. A.: Preparation and characterization of poly(ethylene terephthalate)/clay nanocomposites by melt blending using thermally stable surfactants. Polymers for Advanced Technology, 17, 764-771 (2006). DOI: $10.1002 /$ pat. 752

[7] Yao X., Tian X., Xie D., Zhang X., Zheng K., Xu J., Zhang G., Cui P.: Interface structure of poly(ethylene terephthalate)/silica nanocomposites. Polymer, 50, 1251-1256 (2009).

DOI: 10.1016/j.polymer.2009.01.008

[8] Ke Y-C., Wu T-B., Xia Y-F.: The nucleation, crystallization and dispersion behavior of PET-monodisperse $\mathrm{SiO}_{2}$ composites. Polymer, 48, 3324-3336 (2009).

DOI: 10.1016/j.polymer.2007.03.059

[9] Lee H-J., Oh S-J., Choi J-Y., Kim J. W., Han J., Tan LS., Baek J-B.: In situ synthesis of poly(ethylene terephthalate) (PET) in ethylene glycol containing terephthalic acid and functionalized multiwalled carbon nanotubes (MWNTs) as an approach to MWNT/PET nanocomposites. Chemistry of Matererials, 17, 50575064 (2005). DOI: $10.1021 / \mathrm{cm} 051218 \mathrm{t}$ 
[10] May-Pat A., Avilés F., Toro P., Yazdani-Pedram M., Cauich-Rodríguez J. V.: Mechanical properties of PET composites using multiwalled carbon nanotubes functionalized by inorganic and itaconic acids. Express Polymer Letters, 6, 96-106 (2012).

DOI: $10.3144 /$ expresspolymlett.2012.11

[11] Jin S. H., Park Y-B., Yoon K. H.: Rheological and mechanical properties of surface modified multiwalled carbon nanotube-filled PET composite. Composites Science and Technology, 67, 3434-3441 (2007). DOI: 10.1016/j.compscitech.2007.03.013

[12] Kim H-U., Bang Y. H., Choi S. M., Yoon K. H.: Morphology and mechanical properties of PET by incorporation of amine-polyhedral oligomeric silsesquioxane. Composites Science and Technology, 68, 2739-2747 (2008).

DOI: 10.1016/j.compscitech.2008.05.020

[13] Imai Y., Nishimura S., Abe E., Tateyama H., Abiko A., Yamaguchi A., Aoyama T., Taguchi H.: High-modulus poly(ethylene terephthalate)/expandable fluorine mica nanocomposites with a novel reactive compatibilizer. Chemistry of Matererials, 14, 477-479 (2002).

DOI: $10.1021 / \mathrm{cm} 010408 \mathrm{a}$

[14] Galan E.: Properties and applications of palygorskitesepiolite clays. Clay Minerals, 31, 443-453 (1996).

[15] Murray H. H.: Traditional and new applications for kaolin, smectite, and palygorskite: A general overview. Applied Clay Science, 17, 207-221 (2000).

DOI: $10.1016 / \mathrm{S} 0169-1317(00) 00016-8$

[16] Frost R. L., Mendelovici E.: Modification of fibrous silicates surfaces with organic derivatives: An infrared spectroscopic study. Journal of Colloid and Interface Science, 294, 47-52 (2006).

DOI: $10.1016 /$ j.jcis.2005.07.014

[17] Wang L., Sheng J.: Preparation and properties of polypropylene/org-attapulgite nanocomposites. Polymer, 46, 6243-6249 (2005).

DOI: $10.1016 /$ j.polymer.2005.05.067

[18] Pan H., Chen D.: Preparation and characterization of waterborne polyurethane/attapulgite nanocomposites. European Polymer Journal, 43, 3766-3772 (2007). DOI: 10.1016/j.eurpolymj.2007.06.031

[19] Pan Y., Xu Y., An L., Lu H., Yang Y., Chen W., Nutt S.: Hybrid network structure and mechanical properties of rodlike silicate/cyanate ester nanocomposites. Macromolecules, 41, 9245-9258 (2008).

DOI: $10.1021 / \mathrm{ma} 800819 \mathrm{~s}$

[20] An L., Pan Y., Shen X., Lu H., Yang Y.: Rod-like attapulgite/polyimide nanocomposites with simultaneously improved strength, toughness, thermal stability and related mechanisms. Journal of Material Chemistry, 18, 4928-4941 (2008).

DOI: $10.1039 / \mathrm{B} 805849 \mathrm{~K}$
[21] Yuan X., Li C., Guan G., Liu X., Xiao Y., Zhang D.: Synthesis and characterization of poly(ethylene terephthalate)/attapulgite nanocomposites. Journal of Applied Polymer Science, 103, 1279-1286 (2007).

DOI: $10.1002 / a p p .25207$

[22] Yuan X., Li C., Guan G., Xiao Y., Zhang D.: Thermal stability of surfactants with amino and imido groups in poly(ethylene terephthalate)/clay composites. Journal of Applied Polymer Science, 109, 4112-4120 (2008). DOI: $10.1002 /$ app.28431

[23] Gilman J. W., Awad W. H., Davis R. D., Shields J., Harris R. H., Davis C., Morgan A. B., Sutto T. E., Callahan J., Trulove P. C., DeLong H. C.: Polymer/ layered silicate nanocomposites from thermally stable trialkylimidazolium-treated montmorillonite. Chemistry of Matererials, 14, 3776-3785 (2002).

DOI: $10.1021 / \mathrm{cm} 011532 \mathrm{x}$

[24] Leszczyńska A., Njuguna J., Pielichowski K., Banerjee J. R.: Polymer/montmorillonite nanocomposites with improved thermal properties: Part II. Thermal stability of montmorillonite nanocomposites based on different polymeric matrixes. Thermochimica Acta, 454, 1-22 (2007). DOI: $10.1016 /$ j.tca.2006.11.003

[25] Matayabas J. J. Jr., Turner S. R.: Nanocomposite technology for enhancing the gas barrier of polyetylene terephtalate. in 'Polymer-clay nanocomposites' (eds.: Pinnavaia T. J., Beall G. W.) Wiley, New York, 207228 (2000).

[26] Xu X., Ding Y., Qian Z., Wang F., Wen B., Zhou H., Zhang S., Yang M.: Degradation of poly(ethylene terephthalate)/clay nanocomposites during melt extrusion: Effect of clay catalysis and chain extension. Polymer Degradation and Stability, 94, 113-123 (2009). DOI: $10.1016 /$ j.polymdegradstab.2008.09.009

[27] Calcagno C. I. W., Mariani C. M., Teixeira S. R., Mauler R. S.: The effect of organic modifier of the clay on morphology and crystallization properties of PET nanocomposites. Polymer, 48, 966-974 (2007). DOI: $10.1016 /$ j.polymer.2006.12.044

[28] Chen Z., Luo P., Fu Q.: Preparation and properties of organo-modifier free PET/MMT nanocomposites via monomer intercalation and in situ polymerization. Polymers for Advanced Technology, 20, 916-925 (2009). DOI: $10.1002 /$ pat.1336

[29] Kong Y., Hay J. N.: Multiple melting behaviour of poly(ethylene terephthalate). Polymer, 44, 623-633 (2003).

DOI: $10.1016 / \mathrm{S} 0032-3861(02) 00814-5$

[30] Rao Y. Q., Pochan J. M.: Mechanics of polymer-clay nanocomposites. Macromolecules, 40, 290-296 (2007). DOI: $10.1021 / \mathrm{ma} 061445 \mathrm{w}$ 